\title{
BMJ Open Evaluation of the use of oseltamivir prophylaxis in the control of influenza outbreaks in long-term care facilities in Alberta, Canada: a retrospective provincial database analysis
}

\author{
Ming Ye, ${ }^{1}$ Angela Jacobs, ${ }^{2}$ Muhammad Naeem Khan, ${ }^{2}$ Joy Jaipaul, ${ }^{2}$ Joanna Oda, ${ }^{2}$ \\ Marcia Johnson, ${ }^{2}$ Alexander Doroshenko ${ }^{2,3}$
}

To cite: Ye M, Jacobs A, Khan MN, et al. Evaluation of the use of oseltamivir prophylaxis in the control of influenza outbreaks in longterm care facilities in Alberta, Canada: a retrospective provincial database analysis. BMJ Open 2016;6:e011686. doi:10.1136/bmjopen-2016011686

- Prepublication history and additional material is available. To view please visit the journal (http://dx.doi.org/ 10.1136/bmjopen-2016011686).

Received 26 February 2016 Revised 3 May 2016 Accepted 24 May 2016

CrossMark

\begin{abstract}
${ }^{1}$ School of Public Health, University of Alberta, Edmonton, Alberta, Canada ${ }^{2}$ Alberta Health Services, Edmonton, Alberta, Canada ${ }^{3}$ Faculty of Medicine and Dentistry, Department of Medicine, University of Alberta, Edmonton, Alberta, Canada
\end{abstract}

Correspondence to Dr Alexander Doroshenko; adoroshe@ualberta.ca

\section{ABSTRACT}

Objectives: To evaluate the impact of oseltamivir prophylaxis in the management and control of influenza outbreaks in long-term care facilities in Alberta, Canada.

Setting and participants: Long-term care facilities where 127 influenza outbreaks were reported to public health authorities in Alberta, Canada, during two influenza seasons from 2013 to 2015.

Design and outcome measures: Using routinely collected surveillance and administrative data, we examined the association between decision-making time for oseltamivir recommendation as prophylaxis strategy for influenza outbreaks in long-term care facilities (explanatory variable) and the duration of an influenza outbreak, the postprophylaxis risk of influenza-like illness and hospitalisation among residents of long-term care facilities in Alberta (outcome variables) using multivariable linear and Poisson regression models.

Results: Oseltamivir prophylaxis decision-making time was positively associated with the postintervention duration of an outbreak, with a 1-day delay in making decision on oseltamivir prophylaxis associated with $2.22(95 \% \mathrm{Cl} 1.37$ to 3.06$)$ more days of the duration of an outbreak after controlling for potential confounding effect of the number of residents at risk at intervention, outbreak progression time, prevalence of influenza-like illness during outbreak progression, facility location, presence of mixed strain and based on optimal timing of oseltamivir prophylaxis. Although not statistically significant, a 1-day delay in making decision on oseltamivir prophylaxis was associated with a $5 \%(95 \% \mathrm{Cl}-1 \%$ to $11 \%)$ increase in the postintervention risk of influenza-like illness, and a $6 \%$ $(95 \% \mathrm{Cl}-8 \%$ to $22 \%)$ increase in the postintervention risk of hospitalisation after controlling for the same potential confounders.

Conclusions: Our study demonstrated benefits of using oseltamivir prophylaxis to shorten the duration of influenza outbreaks; however, there were no significant differences in the influenza-like illness and hospitalisation risk occurring after the intervention.

\section{Strengths and limitations of this study}

In a pragmatic evaluation of a public health intervention, we examined whether an association between decision-making time to implement antiviral prophylaxis as a strategy to control influenza outbreaks in long-term care facilities was concordant with the duration of an influenza outbreak and the postprophylaxis risk of influenza-like illness and hospitalisation among facilities residents.

- Our study used province-wide surveillance data in Alberta at the time of influenza outbreaks in long-term care facilities during two consecutive influenza seasons.

- Inclusion of the 2014-2015 influenza season, which was associated with low vaccine effectiveness, allowed us to analyse the effect of oseltamivir antiviral prophylaxis without being confounded by the protective effect of influenza vaccination.

- Our study offers robust methodology for analysing surveillance data and an insight into what variables need to be systematically recorded by surveillance systems pertaining to influenza outbreaks in long-term care facilities.

- This study does not employ intervention-free controls which would not be readily available or recruited in a jurisdiction with universal practice of offering oseltamivir prophylaxis for the control of influenza outbreaks in long-term care facilities.

Surveillance data may offer means of rapid evaluation of oseltamivir prophylaxis in long-term care facilities as a public health measure.

\section{BACKGROUND}

Influenza is a respiratory illness which usually spreads in seasonal epidemics. In 
countries of temperate climate, these epidemics usually occur during winter months. An influenza annual attack rate globally is estimated at $5-10 \%$ in adults and $20-30 \%$ in children. Worldwide, these annual epidemics are estimated to result in about 3-5 million cases of severe illness and about 250 000-500 000 deaths, with most deaths occurring in those older than 65 years. ${ }^{1}$ Influenza results in a significant burden of illness among residents of long-term care facilities (LTCF) as congregate closed settings predispose to rapid viral transmission and frequent outbreaks. ${ }^{2}$ By the end of the 2014-2015 influenza season, 1279 influenza outbreaks in LTCF were reported in Canada. ${ }^{3}$ Similarly, 234 outbreaks with majority occurring in LTCF were observed in the province of Alberta. ${ }^{4}$ Residents in LTCF are at high risk of influenza-related complications resulting in increased morbidity, mortality and hospitalisations. It often leads to a strain on a healthcare system. ${ }^{5}$

Annual influenza vaccination remains the main strategy to prevent influenza illness, including among residents and staff in LTCF. ${ }^{6} 7$ Shortcomings of vaccination include variable uptake, suboptimal vaccine effectiveness (VE) mostly due to mismatch of strains used in the vaccine and circulating strains and poor immune response among the elderly. In the 2014-2015 influenza season, little or no vaccine protection was observed overall, with adjusted $\mathrm{VE}$ against medically attended influenza A (H3N2) infection of $-8 \%(95 \%$ CI $-50 \%$ to $23 \%)$, consistent with vaccine strain mismatch. ${ }^{8}$ Additional strategies to protect residents in LTCF against influenza include non-pharmacological infection control measures (eg, isolation, cohorting, visitation restriction, hand hygiene, wearing masks) and the use of antiviral medications. ${ }^{9}$ Two antiviral agents are currently in use for the treatment and prophylaxis of influenza: oseltamivir in the oral form and zanamivir in the inhalational form. To control influenza outbreaks in closed facilities, the Association of Medical Microbiology and Infectious Disease Canada and the Infectious Diseases Society of America recommend antiviral drug prophylaxis, combined with treatment and influenza vaccine administration, with greater emphasis on antiviral prophylaxis during the 2014/2015 season due to vaccine mismatch. ${ }^{10-12}$ The current practice in Alberta includes using oseltamivir $75 \mathrm{mg}$ once daily for 10 days for antiviral influenza prophylaxis (IP) among non-infected residents when an outbreak of influenza is declared in LTCF. $^{13}$

Evaluation of public health programmes and interventions is an essential organisational practice in public health. ${ }^{14}$ One of the main reasons for programme evaluation is to ensure that effective programmes are maintained and resources are not wasted on ineffective programmes. ${ }^{15}$ A recent cluster randomised controlled study conducted in the Netherlands examined the effectiveness of oseltamivir prophylaxis and found no difference between the intervention and placebo groups for managing influenza outbreaks in LTCF. ${ }^{16}$ Although this study did not have sufficient power, it highlighted the need to conduct evaluations of influenza antiviral prophylaxis interventions in LTCF. The availability and accessibility of comprehensive administrative and surveillance data collected during influenza outbreaks in LTCF in Alberta makes such an evaluation a feasible study which can rapidly provide local context and add to the existing evidence base on this topic. The 2014-2015 influenza season also offers a unique opportunity to examine the effect of oseltamivir IP in the management of outbreaks in LTCF as vaccination is unlikely to have made a material protective effect. The objective of this study is to evaluate the impact of oseltamivir IP in the management and control of influenza outbreaks in LTCF in Alberta using surveillance and administrative data.

\section{METHODS}

We examined the association between the length of time before oseltamivir was recommended as an IP strategy for influenza outbreak control and the duration of an outbreak, the postprophylaxis risk of influenza-like illness (ILI) and hospitalisation among residents in LTCF affected by influenza outbreaks in Alberta during two influenza seasons from 2013 to 2015. The surveillance and administrative data from the Alberta Health Services Communicable Disease/Outbreak Management $(\mathrm{CD} / \mathrm{OM})$ application contained the following data elements for the LTCF influenza outbreaks in Alberta: year of influenza outbreak, location of an outbreak, the outbreak duration, the number of ILI cases and time points for oseltamivir IP interventions and laboratory testing results. ILI was defined as an acute respiratory illness with symptoms such as fever, cough, sore throat, joint pain, muscle aches and severe exhaustion as per the Guidelines for Outbreak Prevention, Control and Management in Acute Care and Facility Living Sites in Alberta and Public Health Agency of Canada case definitions. ${ }^{13} 17$ Detection of influenza virus was confirmed by the Provincial Laboratory for Public Health in Alberta. An outbreak of influenza was defined as having two or more epi-linked cases of ILI within a 7-day period in the same facility, of which at least one was laboratory confirmed. The outbreak was declared over 8 days after onset of the last ILI case in a given facility. ${ }^{12}$ On the same day that an influenza outbreak was confirmed in the LTCF, oseltamivir IP (75 mg daily orally for 10 days) was recommended for all at-risk asymptomatic residents to help control an influenza outbreak. The duration of prophylaxis was extended if the outbreak activity persisted beyond 10 days or discontinued earlier if the outbreak was declared over within 10 days of initiating IP. Symptomatic residents also received a treatment regimen (oseltamivir $75 \mathrm{mg}$ twice daily orally for 5 days). This study was approved by the Health Research Ethics Board of the University of Alberta (study ID Pro00057745). Individual patient consent was not 
required as this study examined de-identified and aggregated surveillance and administrative records collected to monitor an ongoing public health programme.

A series of events occurring during the course of an outbreak in the LTCF are described in figure 1. These include the date that ILI was first reported to Public Health (T1), the date of the second and/or subsequent ILI case(s) reported (also the same date when nasopharyngeal (NP) swabs were sent for laboratory testing) (T2), the date of confirmation of the ILI outbreak as an influenza outbreak (also the same date oseltamivir IP was recommended) (T3) and the date the outbreak was declared over (T4).

The independent (explanatory) variable for this study was decision-making time for oseltamivir IP, which was defined as the length of time between the date when the second or subsequent ILI case(s) were reported (T2) and the date when oseltamivir IP was recommended for implementation in the LTCF (T3) (ie, T3-T2 in figure 1). Variations in this variable reflected the 'reallife' situation in the influenza outbreak management in the LTCF, including the length of time to obtain NP swab results.

The outcome (dependent) variable was the postintervention duration of an outbreak, which was defined as the length of time between when oseltamivir IP was recommended (T3) and the time of declaring outbreak over (ie, T4-T3 in figure 1). Two additional outcome variables of interest were the postintervention risk of new ILI and hospitalisation among LTCF residents (defined as the number of ILI cases and the number of hospitalisations per 100 residents in a given facility after implementing oseltamivir IP). The impact of IP was indirectly inferred: the concordant association would indicate a positive effect of oseltamivir IP (ie, a shorter decision-making time should be positively associated with a shorter outbreak duration and lower ILI and hospitalisation risk), while the discordant association would indicate a negative effect of oseltamivir IP.

The covariates in the analysis included the total number of residents in a given facility, outbreak progression time (T2-T1 in figure 1), the number of ILI cases during outbreak progression time, prevalence of ILI during outbreak progression (ie, the number of ILI cases divided by the number of residents in the T2-T1 period), the number of residents at risk at the start of oseltamivir intervention (T3) (ie, the number of individuals free from ILI at T3) and timing of oseltamivir intervention (ie, the time when oseltamivir IP was recommended in relation to the entire course of an outbreak). As timing of oseltamivir IP intervention (T3) would be related to the postintervention duration of an outbreak (T4-T3) given the same decision-making time (T3-T2) for oseltamivir IP, we generated a timing variable for oseltamivir IP. It was defined as $(\mathrm{T} 3-\mathrm{T} 1) \times 100$ / (T4-T1) to show timing of an intervention, that is, the $i$ th day on which the oseltamivir IP was recommended in a hypothetical 100-day scale of the outbreak.

\section{Statistical analyses}

Differences between time points in figure 1, including oseltamivir IP decision-making time (T3-T2), the postintervention duration of an outbreak (T4-T3) and outbreak progression time (T2-T1), were mathematically calculated and analysed as continuous variables. The location of the LTCF (urban or other), calendar year of

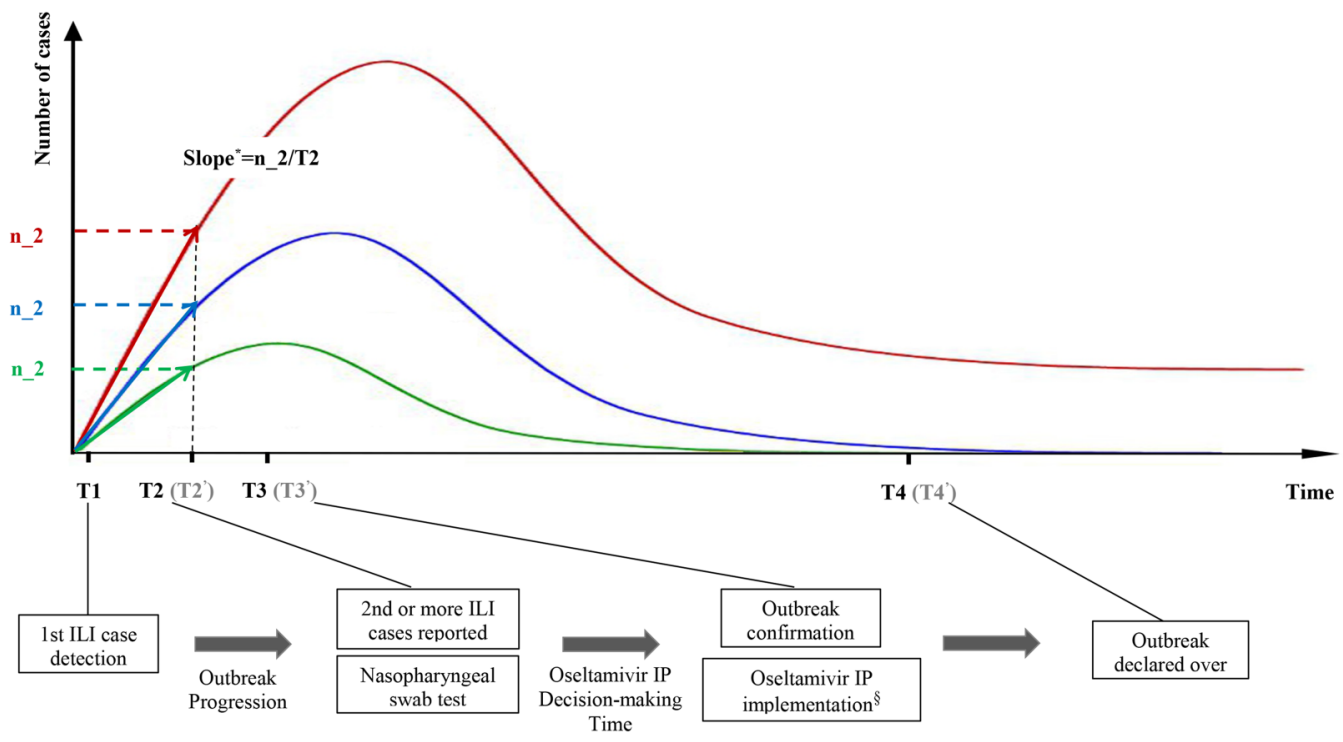

Figure 1 Epidemics and critical events (dates) of influenza outbreaks in the LTCF. *In the slope calculation, number of cases (n) was normalised with total number of residents at LTCF $(\mathrm{N})$, which is equivalent to the prevalence of ILI during outbreak progression. §Timing of oseltamivir intervention $=(T 3-T 1) \times 100 /(T 4-T 1)$, indicating oseltamivir IP occurred on the th day in a hypothetical 100-day scale of the outbreak. A categorical variable was therefore generated based on tertiles of (T3-T1)×100/ (T4-T1) to indicate 'very early/early/late' intervention in relationship with the course of outbreak. ILI, influenza-like illness; IP, influenza prophylaxis; LTCF, long-term care facilities. 
an outbreak, primary strain of influenza during an outbreak (influenza A or B) and whether influenza was mixed with other viruses (mixed or not) were considered as categorical variables. On the basis of the tertiles of continuous variables, including the number of residents at risk at the start of oseltamivir IP (T3), outbreak progression time (T2-T1), prevalence of ILI during outbreak progression, oseltamivir IP decision-making time (T3-T2) and timing of oseltamivir intervention, categorical variables were generated to show gradients in these variables (eg, for time variable-'short/medium/long' and for prevalence variable-'low/medium/high'). In descriptive analyses, means, medians and ranges were calculated for continuous variables and percentages for categorical variables. The postintervention duration of an outbreak was considered as a continuous outcome (dependent) variable. The other two outcome variables, the number of ILI cases and the number of hospitalisations, were considered as count variables following Poisson distribution. For Poisson regression analysis, the number of residents at risk in a given facility was used as an offset to calculate risk of new ILI and hospitalisation after implementation of oseltamivir IP.

Covariates that are potentially related to the postintervention duration (T4-T3) and outcomes (risk of ILI and hospitalisation) of an outbreak, including outbreak progression time (T2-T1), the number of ILI cases during outbreak progression time, prevalence of ILI during outbreak progression, the number of residents at risk at T3 and timing of oseltamivir intervention, were initially examined by the simple linear regression analyses. The means of the postintervention duration of an outbreak (T4-T3) were calculated and compared across different levels of each categorical variable using Student's t-tests or analysis of variance. We checked the normality assumption of the dependent variable (the postintervention duration of an outbreak) using Q-Q plot and kurtosis and Shapiro-Wilk normality tests (see online supplementary figure S1). We further tested whether $\log$ (natural)transformed data would significantly change our results. The postintervention risk of ILI and hospitalisation was calculated and compared across different levels of each categorical variable using Poisson regression analyses. ${ }^{18}$

The magnitude of associations between oseltamivir IP decision-making time (T3-T2) and the duration of an outbreak and the postprophylaxis risk of ILI and hospitalisation were examined using multiple linear and Poisson regression models, after controlling for the confounding effects of the number of residents at risk at intervention (T3), outbreak progression time (T2-T1), prevalence of ILI during outbreak progression, LTCF location, the presence of other respiratory viruses and timing of oseltamivir intervention. Clinically and biologically plausible interactions, such as the effect of year, location, strains of influenza and timing of oseltamivir IP intervention, were examined. Statistical analyses in this study were performed using STATA software (StataCorp LP. 2007, Release V.12, College Station, TX, USA). ${ }^{19}$

\section{RESULTS}

Influenza outbreaks in LTCF in Alberta, 2013-2015

There were 127 influenza outbreaks in 90 LTCF reported in Alberta during 2013/2014-2014/2015 seasons. The characteristics of these outbreaks are summarised in table 1.

During outbreaks, on average, there were 15 ILI cases and one hospitalisation reported per LTCF with the mean risk of ILI of 16.24 per 100 residents (95\% CI $13.0 \%$ to $19.5 \%$ ) and the mean risk of hospitalisation of 1.05 per 100 residents (95\% CI $0.5 \%$ to $1.6 \%$ ). The average decision-making time for oseltamivir IP was 2 days, ranging from 0 to 13 days. Given an average of 17.8 days for the outbreak duration, the timing of oseltamivir IP expressed as tertiles was 3.17, 5.23 and 8.73 days after the detection of the first ILI, for very early, early and late interventions, respectively.

Table 1 Characteristics of long-term care facilities (LTCF) and influenza outbreaks, Alberta, Canada, 2013/2014 and 2014/2015 influenza seasons

\begin{tabular}{|c|c|c|}
\hline Characteristics $(\mathrm{N}=127)$ & $\begin{array}{l}\%, \text { Range, } \\
\text { mean (SE) }\end{array}$ & $95 \% \mathrm{Cl}$ \\
\hline \multicolumn{3}{|c|}{ Number of residents in each LTCF } \\
\hline Range & $9-437$ & _- \\
\hline Average & $92.92(6.28)$ & 80.49 to 105.36 \\
\hline \multicolumn{3}{|l|}{ Location (\%) } \\
\hline Major urban centre & 69.29 & - \\
\hline Other & 30.71 & - \\
\hline \multicolumn{3}{|l|}{ Year of outbreak (\%) } \\
\hline 2013-2014 & 14.17 & - \\
\hline 2014-2015 & 85.83 & \\
\hline \multicolumn{3}{|l|}{ Primary strain (\%) } \\
\hline Influenza A & 79.53 & - \\
\hline Influenza B & 20.47 & - \\
\hline \multicolumn{3}{|l|}{ Mixed strain (\%) } \\
\hline Single & 78.74 & - \\
\hline Mixed & 21.26 & - \\
\hline \multicolumn{3}{|c|}{ Duration of an outbreak (T4-T1: days) } \\
\hline Range & 8-44 & - \\
\hline Average & $17.18(0.51)$ & 16.96 to 18.98 \\
\hline \multicolumn{3}{|c|}{ Outbreak progression time (T2-T1: days) } \\
\hline Range & $0-16$ & - \\
\hline Average & $3.80(0.26)$ & 3.28 to 4.33 \\
\hline \multicolumn{3}{|c|}{$\begin{array}{l}\text { Prevalence of ILI during outbreak progression per } 100 \\
\text { residents }\end{array}$} \\
\hline & $6.95(0.87)$ & 5.22 to 8.67 \\
\hline \multicolumn{3}{|c|}{ Oseltamivir IP decision-making time (T3-T2: days) } \\
\hline Range & $0-13$ & - \\
\hline Average & $1.81(0.16)$ & 1.49 to 2.13 \\
\hline \multicolumn{3}{|c|}{ Postintervention duration of an outbreak (T4-T3: days) } \\
\hline Range & $3-28$ & - \\
\hline Average & $12.35(0.43)$ & 11.50 to 13.21 \\
\hline \multirow{3}{*}{\multicolumn{3}{|c|}{$\begin{array}{l}\text { Overall risk of ILI during an outbreak per } 100 \text { residents } \\
\qquad \begin{array}{l}16.24(1.67) \\
\text { Overall risk of hospitalisation during an outbreak per } 100\end{array} \\
\text { residents }\end{array}$}} \\
\hline & & \\
\hline & & \\
\hline & $1.05(0.29)$ & 0.52 to 1.62 \\
\hline
\end{tabular}


The postprophylaxis outbreak duration and the risk of ILI and hospitalisation in LTCF

The distribution of the postintervention outbreak duration (T4-T3) by outbreak characteristics is shown in table 2.

The average postintervention duration of an outbreak was longer in the LTCF located in major urban centres in comparison to other zones in Alberta. Influenza outbreaks associated with other respiratory viruses (mixed strains) had a longer postintervention duration compared with the outbreaks with influenza viruses only. Outbreaks with a rapid progression (ie, an average of 1.2 days in outbreak progression time), had a longer postintervention duration of an outbreak (13.8 vs 11.1 vs 11.8 days, respectively) compared with the outbreaks with medium (3.4 days) and long (7.2 days) outbreak progression times. Although the unadjusted comparisons showed a negative association between prevalence of ILI during outbreak progression time and the postintervention duration of an outbreak, the magnitude of this association was close to null and not statistically significant in the multivariable regression analyses (see online supplementary table S1a). Using log-transformed data in linear regression analysis resulted in a 1.16 times longer duration of the postintervention duration of an outbreak for a 1-day delay in oseltamivir IP. This association was statistically significant and similarly effect modified by the timing of the intervention as was the model using non-transformed data (see online supplementary table S1b).

The distribution of the postintervention risk of ILI and hospitalisation across influenza outbreak variables and characteristics of LTCF are summarised in table 3 .

The postintervention risk of ILI was significantly lower in the LTCF located in major urban centres than in other areas in Alberta. The influenza A outbreaks and influenza outbreaks mixed with other respiratory viruses were associated with a higher postintervention risk of ILI compared with influenza B outbreaks and influenza only (not mixed) outbreaks. While there was no significant difference across outbreak progression time

Table 2 The unadjusted postintervention duration of an outbreak in long-term care facilities affected by influenza outbreaks in Alberta, Canada, 2013/2014 and 2014/2015 influenza seasons

\begin{tabular}{|c|c|c|c|}
\hline Characteristics ( $\mathrm{N}=127)$ & Mean (SE) (days) & $95 \% \mathrm{Cl}$ (days) & p Value \\
\hline \multicolumn{4}{|l|}{ Average } \\
\hline & $12.35(0.43)$ & 11.50 to 13.21 & - \\
\hline \multicolumn{4}{|l|}{ Location } \\
\hline Major urban centre & $13.08(0.50)$ & 12.09 to 14.07 & - \\
\hline Other & $10.72(0.79)$ & 9.16 to 12.28 & 0.011 \\
\hline \multicolumn{4}{|l|}{ Year } \\
\hline 2013-2014 & $13.61(1.22)$ & 11.20 to 16.02 & - \\
\hline 2014-2015 & $12.15(0.46)$ & 11.24 to 13.06 & 0.238 \\
\hline \multicolumn{4}{|l|}{ Primary strain } \\
\hline Influenza A & $12.38(0.45)$ & 11.48 to 13.27 & - \\
\hline Influenza B & $12.27(1.19)$ & 9.91 to 14.63 & 0.921 \\
\hline \multicolumn{4}{|l|}{ Mixed strain } \\
\hline Single & $11.56(0.44)$ & 10.68 to 12.44 & - \\
\hline Mixed & $15.30(1.03)$ & 13.25 to 17.34 & $<0.0001$ \\
\hline \multicolumn{4}{|c|}{ Outbreak progression time (T2-T1) } \\
\hline Short & $13.83(0.74)$ & 12.37 to 15.29 & - \\
\hline Medium & $11.13(0.70)$ & 9.74 to 12.51 & 0.009 \\
\hline Long & $11.85(0.75)$ & 10.37 to 13.33 & 0.055 \\
\hline \multicolumn{4}{|c|}{ Prevalence of ILI during outbreak progression } \\
\hline Low & $13.43(0.67)$ & 12.11 to 14.76 & - \\
\hline Medium & $12.59(0.75)$ & 11.11 to 14.06 & 0.419 \\
\hline High & $10.95(0.79)$ & 9.38 to 12.52 & 0.017 \\
\hline \multicolumn{4}{|c|}{ Oseltamivir IP decision-making time (T3-T2) } \\
\hline Short & $11.72(0.63)$ & 10.46 to 12.97 & - \\
\hline Medium & $13.36(0.63)$ & 12.12 to 14.61 & 0.111 \\
\hline Long & $12.42(1.03)$ & 10.37 to 14.47 & 0.514 \\
\hline \multicolumn{4}{|c|}{ Timing of oseltamivir intervention } \\
\hline Very early in outbreak & $15.30(0.73)$ & 13.86 to 16.75 & - \\
\hline Early in outbreak & $12.62(0.64)$ & 11.36 to 13.89 & 0.004 \\
\hline Late in outbreak & $9.07(0.53)$ & 8.03 to 10.12 & $<0.0001$ \\
\hline \multicolumn{4}{|c|}{ Number of residents at risk at intervention } \\
\hline 1st tertile & $9.47(0.63)$ & 8.23 to 10.71 & - \\
\hline 2nd tertile & $12.58(0.65)$ & 11.30 to 13.85 & 0.001 \\
\hline 3rd tertile & $15.24(0.69)$ & 13.87 to 16.61 & $<0.0001$ \\
\hline
\end{tabular}


Table 3 The unadjusted postintervention risk of influenza-like illness and hospitalisation in long-term care facilities affected by influenza outbreaks in Alberta, Canada, 2013/2014 and 2014/2015 influenza seasons

\begin{tabular}{|c|c|c|c|c|c|c|}
\hline \multirow[b]{2}{*}{ Characteristics $(\mathrm{N}=127)$} & \multicolumn{3}{|l|}{ ILI } & \multicolumn{3}{|l|}{ Hospitalisation } \\
\hline & $\begin{array}{l}\text { Mean (SE) } \\
\text { (per } 100 \text { residents) }\end{array}$ & $95 \% \mathrm{Cl}$ & p Value & $\begin{array}{l}\text { Mean (SE) } \\
\text { (per } 100 \text { residents) }\end{array}$ & $95 \% \mathrm{Cl}$ & p Value \\
\hline \multicolumn{7}{|l|}{ Average } \\
\hline & $6.60(1.27)$ & 4.19 to 9.10 & - & $1.04(0.26)$ & 0.52 to 1.55 & - \\
\hline \multicolumn{7}{|l|}{ Location } \\
\hline Major urban centre & $6.09(1.29)$ & 3.56 to 8.59 & - & $1.00(0.28)$ & 0.45 to 1.55 & - \\
\hline Other & $9.33(2.88)$ & 3.72 to 14.91 & $<0.0001$ & $1.31(0.71)$ & 0.01 to 2.73 & 0.205 \\
\hline \multicolumn{7}{|l|}{ Year } \\
\hline 2013-2014 & $5.92(5.34)$ & 0.01 to 16.41 & - & $1.03(1.14)$ & 0.001 to 3.31 & - \\
\hline 2014-2015 & $6.75(1.20)$ & 4.40 to 9.15 & 0.212 & $1.06(0.28)$ & 0.51 to 1.60 & 0.912 \\
\hline \multicolumn{7}{|l|}{ Primary strain } \\
\hline Influenza A & $7.13(1.55)$ & 4.09 to 10.15 & - & $1.16(0.36)$ & 0.45 to 1.86 & - \\
\hline Influenza B & $5.14(0.82)$ & 3.52 to 6.79 & $<0.0001$ & $0.73(0.23)$ & 0.30 to 1.18 & 0.046 \\
\hline \multicolumn{7}{|l|}{ Mixed strain } \\
\hline Single & $6.15(1.21)$ & 3.79 to 8.52 & - & $1.14(0.31)$ & 0.53 to 1.76 & - \\
\hline Mixed & 7.76 (3.92) & 0.08 to 15.41 & 0.004 & $0.82(0.77)$ & 0.002 to 2.33 & 0.139 \\
\hline \multicolumn{7}{|c|}{ Outbreak progression time (T2-T1) } \\
\hline Short & $6.34(1.11)$ & 4.15 to 8.51 & - & $0.73(0.25)$ & 0.24 to 1.22 & - \\
\hline Medium & $6.69(3.23)$ & 0.36 to 13.02 & 0.572 & $1.18(0.57)$ & 0.06 to 2.31 & 0.041 \\
\hline Long & $6.83(2.02)$ & 2.86 to 10.74 & 0.404 & $1.30(0.66)$ & 0.006 to 2.60 & 0.01 \\
\hline \multicolumn{7}{|c|}{ Prevalence of ILI during outbreak progression } \\
\hline Low & $4.86(0.66)$ & 3.58 to 6.11 & - & $0.52(0.13)$ & 0.27 to 0.78 & - \\
\hline Medium & $7.08(1.81)$ & 3.53 to 10.61 & $<0.0001$ & $1.33(0.41)$ & 0.53 to 2.14 & $<0.0001$ \\
\hline High & $11.69(3.26)$ & 5.32 to 18.11 & $<0.0001$ & $2.01(0.76)$ & 0.53 to 3.50 & $<0.0001$ \\
\hline \multicolumn{7}{|c|}{ Oseltamivir IP decision-making time (T3-T2) } \\
\hline Short & $7.68(1.87)$ & 4.01 to 11.33 & - & $1.17(0.47)$ & 0.25 to 2.08 & - \\
\hline Medium & $6.13(2.78)$ & 0.69 to 11.58 & 0.008 & $1.05(0.59)$ & 0.006 to 2.22 & 0.607 \\
\hline Long & $5.44(1.89)$ & 1.75 to 9.14 & 0.001 & $0.84(0.35)$ & 0.15 to 1.53 & 0.191 \\
\hline \multicolumn{7}{|c|}{ Timing of oseltamivir intervention } \\
\hline Very early in outbreak & $6.85(1.51)$ & 3.85 to 9.81 & - & $0.79(0.26)$ & 0.28 to 1.30 & - \\
\hline Early in outbreak & $8.43(2.90)$ & 2.74 to 11.14 & 0.011 & $1.35(0.64)$ & 0.10 to 2.61 & 0.012 \\
\hline Late in outbreak & $3.61(1.80)$ & 0.08 to 7.15 & $<0.0001$ & $1.08(0.54)$ & 0.02 to 2.14 & 0.20 \\
\hline \multicolumn{7}{|c|}{ Number of residents at risk at intervention } \\
\hline 1st tertile & $9.77(3.32)$ & 3.27 to 16.27 & - & $2.13(0.76)$ & 0.64 to 3.63 & - \\
\hline 2nd tertile & $7.39(0.95)$ & 5.53 to 9.27 & 0.022 & $0.68(0.18)$ & 0.33 to 1.03 & $<0.0001$ \\
\hline 3rd tertile & $5.80(0.90)$ & 4.40 to 7.56 & $<0.0001$ & $1.00(0.20)$ & 0.58 to 1.38 & 0.001 \\
\hline
\end{tabular}

(T2-T1), higher prevalence of ILI during outbreak progression was associated with an increased postintervention risk of ILI.

Similar to the risk of ILI, an outbreak associated with influenza A had a higher postintervention risk of hospitalisation compared with influenza B outbreaks. A longer outbreak progression time (T2-T1) and higher prevalence of ILI during outbreak progression were associated with a higher postintervention risk of hospitalisation after oseltamivir IP.

\section{An impact of oseltamivir prophylaxis decision-making}

time on the outbreak duration and the risk of ILI and hospitalisation in LTCF

In multiple linear regression analyses, oseltamivir IP decision-making time (T3-T2) was positively associated with the postintervention duration of an outbreak (T4-T3), with a 1-day delay in making decision on oseltamivir IP resulting in 2.22 (95\% CI 1.37 to 3.06) more days of the outbreak duration $(\mathrm{p}<0.0001$; table 4$)$ after controlling for the number of residents at risk at intervention (T3), outbreak progression time (T2-T1), prevalence of ILI during outbreak progression, location, presence of mixed respiratory viruses and based on optimal timing of oseltamivir IP intervention. Timing of oseltamivir IP intervention in the course of the outbreak displayed effect modification on oseltamivir IP decisionmaking time, with an early intervention associated with a stronger impact on the postintervention duration of an outbreak. For very early (1st tertile), early (2nd tertile) and late (3rd tertile) timing of oseltamivir IP intervention, a 1-day delay in making decision on oseltamivir IP was associated with 2.22 (95\% CI 1.37 to 3.06$), 1.31$ (95\% CI 0.71 to 1.91$)$ and 0.86 (95\% CI 0.42 to 1.30$)$ more days of the outbreak duration, respectively $(\mathrm{p}<0.0001$; table 4). Although not statistically significant, in the 
Table 4 The adjusted associations between oseltamivir IP decision-making time and the postintervention duration of an outbreak and the risk of ILI and hospitalisation in LTCF affected by influenza outbreaks in Alberta, Canada, 2013/2014 and 2014/2015 influenza seasons

\begin{tabular}{|c|c|c|c|}
\hline \multirow[b]{2}{*}{ Characteristics $(\mathrm{N}=127)$} & \multicolumn{3}{|c|}{ Postintervention duration of an outbreak } \\
\hline & $\overline{\boldsymbol{\beta}^{\star}}$ & $95 \% \mathrm{Cl}$ & p Value \\
\hline \multicolumn{4}{|c|}{ Oseltamivir IP decision-making time by timing of oseltamivir intervention } \\
\hline Very early in outbreak & 2.22 & 1.37 to 3.06 & $<0.0001$ \\
\hline Early in outbreak & 1.31 & 0.71 to 1.91 & $<0.0001$ \\
\hline \multirow[t]{3}{*}{ Late in outbreak } & 0.86 & 0.42 to 1.30 & $<0.0001$ \\
\hline & \multicolumn{3}{|c|}{ Postintervention ILI risk } \\
\hline & $\mathbf{R R}^{*}$ & $95 \% \mathrm{Cl}$ & p Value \\
\hline \multirow[t]{3}{*}{ Oseltamivir IP decision-making time } & 1.05 & 0.99 to 1.11 & 0.121 \\
\hline & \multicolumn{3}{|c|}{ Postintervention hospitalisation risk } \\
\hline & $\mathbf{R R}^{*}$ & $95 \% \mathrm{Cl}$ & p Value \\
\hline Oseltamivir IP decision-making time & 1.06 & 0.92 to 1.22 & 0.418 \\
\hline
\end{tabular}

multiple Poisson regression, a 1-day delay in making decision on oseltamivir IP (T3-T2) was associated with a $5 \%$ (95\% CI $-1 \%$ to $11 \%)$ increase in the postintervention risk of ILI, and a $6 \%(95 \%$ CI $-8 \%$ to $22 \%)$ increase in the postintervention risk of hospitalisation after controlling for same potential confounders described above (table 4 and online supplementary tables S2 and S3). No significant interactions were found between oseltamivir IP decision-making time and influenza season, location, strains of influenza and timing of intervention.

\section{DISCUSSION}

Our study adds to the discussion on the effectiveness of oseltamivir IP in LTCF. We demonstrated that timely use of oseltamivir IP in the management of influenza outbreaks in LTCF has benefits on shortening the outbreak duration, but has only modest impact on the number of cases of ILI and hospitalisations averted. Our results highlight that any delay in the administration of oseltamivir IP leads to a longer outbreak duration; the earlier this recommendation is implemented, the more pronounced the effect on shortening of the outbreak duration. In our study, the mean delay in recommending oseltamivir IP is 1.81 days. On the basis of our regression analysis, comparing LTCF with a 0-day delay in antiviral prophylaxis to those with an average delay, there will be almost 4 fewer days in the outbreak duration and a $9 \%[\exp (\ln (1.05) \times 1.81)-1]$ decrease in the risk of ILI. Furthermore, for the postintervention duration of an outbreak in our study, results from nontransformed data were comparable with the model using log-transformed data in linear regression, thus supporting our conclusions. For example, $\beta$ coefficient of 2.22 days from the non-transformed model was equivalent to $18 \% \quad((12.4+2.22) / 12.4=1.18)$ increase (16\% in log-transformed model) in the duration of an outbreak per 1-day delay in instituting IP, given the mean post IP outbreak duration of 12.4 days in this study. We however found less evident effect of oseltamivir IP on ILI cases and hospitalisations averted versus on the duration of an outbreak. This could be due to significant variations in identification of ILI cases in the LTCF compared to definitions of outbreaks; however, there also could be a difference in timing for declaring an outbreak over, depending on whether that date falls on a weekday or weekend (eg, an outbreak may be formally closed the next business day after the weekend even if criteria for the outbreak cessation were met earlier). We also found geographical differences between urban and rural regions in terms of ILI risk and the outbreak duration. In adjusted analysis, regardless of the effect of oseltamivir IP and other variables, the duration of an outbreak and the risk of ILI were greater in rural regions in comparison to urban areas.

Current evidence base addressing the use of oseltamivir as a prophylactic agent in LTCF include a limited number of randomised controlled trials (RCTs). van der Sande et al conducted a cluster randomised, placebocontrolled trial in the Netherlands in 60 LTCF over four influenza seasons from 2009 to 2013 and found no statistical difference between IP intervention with oseltamivir versus placebo on incidence of new confirmed influenza cases and ILI cases after initiation of IP. This study did not have sufficient power because only a small number of outbreaks occurred during the study period. ${ }^{16}$ Booy et $a t^{20}$ compared the effects of treatment alone with combined treatment and IP in another cluster RCT among age-care facilities in Australia in 2006-2008 and reported a statistically significant difference between groups for 
influenza attack rates and the outbreaks' duration, but no difference for deaths, hospitalisations and pneumonia. Strategies of using oseltamivir prophylaxis in all nursing home $(\mathrm{NH})$ residents versus only among those directly exposed residents versus no prophylaxis were examined in a non-randomised controlled study in Slovenia during 2011/2012 influenza outbreak. Results were mixed. The proportion of hospitalisations and deaths among $\mathrm{NH}$ residents was the highest in the 'no-prophylaxis' group suggesting benefits of antiviral prophylaxis; however, the proportion of acute respiratory illness was also the lowest in the 'no-prophylaxis' group. The duration of outbreaks was the shortest in the universal prophylaxis group. Hospitalisations and deaths were non-influenza specific. $^{21}$ Findings from a number of observational and descriptive studies generally favour the recommendation of using antiviral IP to control outbreaks of influenza in the LTCF. ${ }^{22-26}$ These studies ranged from case series to uncontrolled cohort studies, before-and-after design, outbreak reports and varied in the sample size, degree and scope of analyses undertaken. In comparison with the above reports, our results were similar to findings by van der Sande et al for the ILI risk outcome, but on the other hand demonstrated benefits of oseltamivir IP on shortening the duration of outbreaks reported in some controlled and observational studies. The question remains what is the optimal outcome measure to evaluate the effect of oseltamivir IP in LTCF. The postintervention laboratory-confirmed influenza risk likely represents the most objective outcome measure; however, it is likely to be unavailable outside of controlled settings, as is in our study. It is a standard practice in Alberta not to test all individuals in LTCF once an outbreak of influenza is laboratory confirmed and declared. Individuals developing ILI are assumed to have influenza in the outbreak setting. Our study has strengths and limitations. The strength of our study is that it used province-wide surveillance and administrative data at the time of outbreaks. Analysis of province-wide data minimised the possibility of selection bias in our study. We analysed multiple time points on the continuum from the first ILI case to declaring outbreak over, geographical variations, types of outbreaks by pathogen and examined different influenza seasons. The latter is particularly important as the 2014-2015 influenza season was associated with low $\mathrm{VE}$, and therefore the effect of oseltamivir IP was unlikely to be confounded by influenza vaccination. As our study was performed in the jurisdiction where oseltamivir IP is routinely recommended to manage all confirmed influenza outbreaks in the LTCF, no intervention-free controls were readily available. We therefore analysed the timeliness in recommending oseltamivir IP and measured the effects of oseltamivir IP decision-making time. This approach represents a pragmatic 'real-life' evaluation of a public health intervention and can complement findings from randomised trials. Furthermore, we used time to recommend antiviral prophylaxis in determining decision- making time rather than the actual administration of oseltamivir. This represents a similar concept to the 'intention-to-treat' analysis used in RCTs.

Limitations include the fact that we did not use laboratory-confirmed influenza cases as an outcome measure as residents in the LTCF are not routinely tested for influenza when a confirmed influenza outbreak is underway. We used ILI in the context of a confirmed influenza outbreak as a proxy for influenza cases. However, the effect of substituting the risk of ILI as an outcome variable for laboratory-confirmed cases of influenza on conclusions of our study is difficult to predict. Also, temporal hospitalisations recorded in the administrative database were not specific to influenza. It is also plausible that different LTCF could have different resident population (eg, some LTCF may house residents with more comorbidities). Furthermore, it is possible that LTCF that implement oseltamivir prophylaxis early on are also the ones which may also implement better outbreak detection systems, have better trained staff and be more effective in implementing other control measures such as isolation, hand and environmental hygiene. However, we would expect less heterogeneity between LTCF given that Alberta Health Services employs several strategies to facilitate standardisation of outbreak management in LTCF. Strategies include publishing guidelines on outbreak management in LTCF which are generally well adhered to province wide, providing LTCF with annual outbreak management education and updates through collaboration between stakeholders involved in outbreak management, facilitating postinfluenza season debriefs and supporting targeted immunisation programmes in LTCF.

The rarity of randomised controlled studies specifically conducted in LTCF underlines the challenges for making evidence-based recommendations in these settings. Randomised controlled studies may require substantial resources and due to the unpredictability of outbreaks may turn out to be inconclusive as evidenced by the van der Sande study. In these circumstances, the role of rapidly analysing existing surveillance and administrative data should be emphasised. Our study offers an insight into what variables need to be systematically recorded by surveillance systems pertaining to influenza outbreaks in LTCF and robust methodology for analysing such data. Such analyses can be performed rapidly and, while not a substitute to RCTs, are more feasible to conduct and substantially less costly. Should results be reproducible, this will add to the evidence base on the topic. Our results are likely to be transferable to other jurisdictions in Canada and other developed countries which use the same policies for LTCF influenza outbreak detection, reporting and management.

\section{CONCLUSION}

This study provides evidence in Alberta where using oseltamivir IP is recommended and where no 
intervention-free controls are readily available. On the basis of the analysis of routinely collected administrative and surveillance data, it demonstrated the benefits of using oseltamivir IP to shorten the duration of influenza outbreaks; however, there was no significant effect on the risk of ILI and hospitalisation occurring after the intervention. Study results may be used to inform policy and guidelines for use of oseltamivir IP in influenza outbreak management and control in LTCF.

Acknowledgements The authors would like to acknowledge the staff at the participating long-term care facilities for their contribution in an ongoing data reporting and collection as well as to Alberta Health Services staff managing the Communicable Disease/Outbreak Management (CD/OM) application. The authors also thank members of the Alberta Influenza Steering Committee for their comments.

Contributors AD conceived an idea, originated the study and together with MY designed the study methodology. MY conducted all statistical analyses on data. $A D$ and $M Y$ jointly wrote the manuscript. MNK extracted and prepared relevant data from the CDOM application. $\mathrm{AJ}, \mathrm{JJ}, \mathrm{JO}$ and $\mathrm{MJ}$ contributed to the study design and defining data variables. All authors revised the manuscript for intellectual content and approved final submission.

Funding This work was supported by Alberta Health, grant number RES0025110. Competing interests None declared.

Ethics approval This study was approved by the Health Research Ethics Board of the University of Alberta (study ID Pro00057745).

Provenance and peer review Not commissioned; externally peer reviewed.

Data sharing statement No additional data are available.

Open Access This is an Open Access article distributed in accordance with the Creative Commons Attribution Non Commercial (CC BY-NC 4.0) license, which permits others to distribute, remix, adapt, build upon this work noncommercially, and license their derivative works on different terms, provided the original work is properly cited and the use is non-commercial. See: http:// creativecommons.org/licenses/by-nc/4.0/

\section{REFERENCES}

1. World Health Organization. Seasonal Influenza Factsheet. http:// www.who.int/mediacentre/factsheets/fs211/en/ (accessed Feb 2016).

2. Bradley SF. Prevention of influenza in long-term-care facilities. Long-term-care committee of the society for healthcare epidemiology of America. Infect Control Hosp Epidemiol 1999;20:629-37.

3. Public Health Agency of Canada. FluWatch report: May 26 to June, 62015 (Weeks 21 and 22). http://www.phac-aspc.gc.ca/fluwatch/ 14-15/w22_15/assets/pdf/fw2015-22-eng.pdf (accessed Feb 2016).

4. Alberta Health. 2014/15 Influenza Surveillance Report Week 15, April 5-18, 2015. http://www.health.alberta.ca/documents/ Influenza-Surveillance-2015-15.pdf (accessed Feb 2016).

5. Carroll NV, Delafuente JC, McClure KL, et al. Economic burden of influenza-like illness in long-term-care facilities. Am J Health Syst Pharm 2001:58:1133-8.

6. Wendelboe AM, Avery C, Andrade B, et al. Importance of employee vaccination against influenza in preventing cases in long-term care facilities. Infect Control Hosp Epidemiol 2011;32:990-7.
7. Person CJ, Nadeau JA, Schaffzin JK, et al. Influenza immunization coverage of residents and employees of long-term care facilities in New York State, 2000-2010. Am J Infect Control 2013;41:743-5.

8. Skowronski DM, Chambers C, Sabaiduc $S$, et al. Interim estimates of 2014/2015 vaccine effectiveness against influenza A (H3N2) from Canada's sentinel physician surveillance Network, January 2015. Euro Surveill 2015;20:pii: 21022.

9. Rainwater-Lovett K, Chun K, Lessler J. Influenza outbreak control practices and the effectiveness of interventions in long-term care facilities: a systematic review. Influenza Other Respir Viruses 2014;8:74-82.

10. Aoki FY, Allen UD, Stiver HG, et al. Guidance for practitioners on the use of antiviral drugs to control influenza outbreaks in long-term care facilities in Canada, 2014-2015 season. Can J Infect Dis Med Microbiol 2015;26:e1-4

11. Aoki FY, Allen UD, Stiver HG, et al. The use of antiviral drugs for influenza: guidance for practitioners 2012/2013. Can J Infect Dis Med Microbiol 2012;23:e79-92.

12. Harper SA, Bradley JS, Englund JA, et al. Seasonal influenza in adults and children--diagnosis, treatment, chemoprophylaxis, and institutional outbreak management: clinical practice guidelines of the Infectious Diseases Society of America. Clin Infect Dis 2009;48:1003-32.

13. Alberta Health Services. Guidelines for Outbreak Prevention, Control and Management in Acute Care and Facility Living Sites. http://www. albertahealthservices.ca/assets/healthinfo/Diseases/hi-dis-flu-provhlsl.pdf (accessed Feb 2016).

14. Dyal WW. Ten organizational practices of public health: a historical perspective. Am J Prev Med 1995;11(Suppl 6):6-8.

15. Centers for Disease Control and Prevention. Introduction to Program Evaluation for Public Health Programs: A Self-Study Guide. http:// www.cdc.gov/eval/guide/introduction/index.htm (accessed Feb 2016).

16. van der Sande MA, Meijer A, Sen-Kerpiclik F, et al. Effectiveness of post-exposition prophylaxis with oseltamivir in nursing homes: a randomised controlled trial over four seasons. Emerg Themes Epidemiol 2014;11:13.

17. Public Health Agency of Canada. FluWatch.Definitions for 20142015 season. http://www.phac-aspc.gc.ca/fluwatch/14-15/ def14-15-eng.php (accessed Feb 2016).

18. Armitage P, Berry G, Matthews JNS. Statistical methods in medical research. John Wiley and Sons, 2008.

19. Juul S, Frydenberg M. An introduction to Stata for health researchers. Taylor \& Francis, 2010.

20. Booy R, Lindley RI, Dwyer DE, et al. Treating and preventing influenza in aged care facilities: a cluster randomised controlled trial. PLOS ONE 2012;7:e46509.

21. Gorišek Miksić N, Uršič T, Simonović Z, et alOseltamivir prophylaxis in controlling influenza outbreak in nursing homes: a comparison between three different approaches. Infection 2015;43:73-81.

22. Bowles SK, Lee W, Simor AE, et al. Use of oseltamivir during influenza outbreaks in Ontario Nursing Homes, 1999-2000. J Am Geriatr Soc 2002;50:608-16.

23. Parker R, Loewen N, Skowronski D. Experience with oseltamivir in the control of a nursing home influenza B outbreak. Can Commun Dis Rep 2001;27:37-40.

24. Seale $\mathrm{H}$, Weston $\mathrm{KM}$, Dwyer DE, et alThe use of oseltamivir during an influenza B outbreak in a chronic care hospital. Influenza Other Respir Viruses 2009;3:15-20.

25. Monto AS, Rotthoff J, Teich E, et al. Detection and control of influenza outbreaks in well-vaccinated nursing home populations. Clin Infect Dis 2004;39:459-64.

26. Chang YM, Li WC, Huang CT, et al. Use of oseltamivir during an outbreak of influenza $A$ in a long-term care facility in Taiwan. J Hosp Infect 2008:68:83-7. 